Review Article

\title{
Efficacy of Lianhua Qingwen Compared with Conventional Drugs in the Treatment of Common Pneumonia and COVID-19 Pneumonia: A Meta-Analysis
}

\author{
Caiyun Hu, ${ }^{1}$ Mingming Liang, ${ }^{2}$ Fengfeng Gong, ${ }^{3}$ Bin He, ${ }^{1}$ Dongdong Zhao, ${ }^{4}$ \\ and Guoliang Zhang ${ }^{1}{ }^{1}$ \\ ${ }^{1}$ Department of Scientific Research, The First Affiliated Hospital of Anhui University of Traditional Chinese Medicine, \\ 117 Meishan Road, Hefei, Anhui, China \\ ${ }^{2}$ Epidemiology and Health Statistics, School of Public Health, Anhui Medical University, No. 81 Meishan Road, Hefei, \\ Anhui, China \\ ${ }^{3}$ Fuyang Hospital of Anhui Medical University, No. 99 Huangshan Road, Fuyang, Anhui, China \\ ${ }^{4}$ Infection Department, The First Affiliated Hospital of Anhui Medical University, No. 218 Jixi Road, Hefei, Anhui, China \\ Correspondence should be addressed to Guoliang Zhang; zhangguoliang61@sina.com
}

Received 15 May 2020; Revised 19 August 2020; Accepted 9 September 2020; Published 18 September 2020

Academic Editor: Daniela Rigano

Copyright (c) 2020 Caiyun Hu et al. This is an open access article distributed under the Creative Commons Attribution License, which permits unrestricted use, distribution, and reproduction in any medium, provided the original work is properly cited.

\begin{abstract}
Background and Objectives. Lianhua Qingwen is a traditional Chinese medicine, which has been reported to be effective in the treatment of pneumonia. However, no consensus has been reached; therefore, we aimed to provide reliable assessments of common pneumonia and COVID-19 pneumonia. Methods. Seven English and Chinese databases were used to search for qualified experimental studies as of July 27, 2020. All data were extracted directly from the included studies, and no special conversion formula was used. The weighted mean difference (WMD), 95\% confidence interval (CI), and odds ratio (OR) were used for evaluation. Results. Forty-two studies involving 3793 subjects met the qualification criteria. For common pneumonia, a short duration of flu-like symptoms (WMD $=-1.81,95 \% \mathrm{CI}=-2.12$ to $-1.50, P<0.001$ ), sputum (WMD $=-1.10,95 \% \mathrm{CI}=-1.50$ to $-0.70, P<0.001$ ), pulmonary rale $(\mathrm{WMD}=-2.03,95 \% \mathrm{CI}=-2.74$ to $-1.31, P<0.001$ ), pulmonary imaging improvement (WMD $=-1.88,95 \% \mathrm{CI}=-2.28$ to $-1.47, P<0.001)$, curative effect $(\mathrm{OR}=3.65,95 \% \mathrm{CI}=2.81$ to $4.76, P<0.001)$, and healing period $(\mathrm{WMD}=-1.68,95 \% \mathrm{CI}=-2.62$ to $-0.74, P<0.001)$ were associated with the Lianhua Qingwen group; subgroup analysis based on flu-like symptoms showed statistically significant improvements in fever and cough. For COVID-19 pneumonia, improvements in flu-like symptoms $(\mathrm{OR}=3.18,95 \% \mathrm{CI}=2.36$ to $4.29, P<0.001)$, shortness of breath $(\mathrm{OR}=10.62,95 \% \mathrm{CI}=3.71$ to $30.40, P<0.001)$, curative effect $(\mathrm{OR}=2.49,95 \% \mathrm{CI}=1.76$ to $3.53, P<0.001)$, healing period $(\mathrm{WMD}=-2.06,95 \% \mathrm{CI}=-3.36$ to $-0.75, P=0.002)$, and conversion of severe cases $(\mathrm{OR}=0.46,95 \% \mathrm{CI}=0.27$ to $0.77, P=0.003)$ were associated with the Lianhua Qingwen group; subgroup analysis indicated statistically significant improvements of fever, cough, fatigue, and muscle pain in the Lianhua Qingwen group compared to the conventional drug group. Regarding adverse reactions, no significant difference was detected for common pneumonia $(\mathrm{OR}=0.75,95 \% \mathrm{CI}=0.54$ to $1.05, P=0.097)$. Conclusions. Lianhua Qingwen combined with conventional drugs may be a promising therapy for treating common pneumonia and COVID-19 pneumonia.
\end{abstract}

\section{Introduction}

Traditional Chinese medicine, with a thousand years of history in China, has been used to treat various diseases [1-5]. Lianhua Qingwen (连花清瘟), which is obtained by the reduction and addition of combined Maxing Shigan decoction and Yin Qiao powder, has been widely accepted as a broad-spectrum antiviral agent in the clinic [6]. It consists of 13 herbs: Lian Qiao (Fructus Forsythiae, weeping forsythia capsule, 连趐), $255 \mathrm{~g}$; Ma Huang (Herba Ephedrae, ephedra, 麻黄), $85 \mathrm{~g}$; Jin Yin Hua (Flos Lonicerae, honeysuckle flower, 金银花), $255 \mathrm{~g}$; Ban Lan Gen (Radix Isatidis, 
isatis root, 板蓝根), $255 \mathrm{~g}$; Mianma Guanzhong (Rhizoma Dryopteris crassirhizomae, male fern rhizome, 绵马贯众), $255 \mathrm{~g}$; Bo He (Herba Menthae, menthol, 薄荷), $7.5 \mathrm{~g}$; Shi Gao (Gypsum Fibrosum, gypsum, 石膏), $255 \mathrm{~g}$; Guang Huo Xiang (Herba Pogostemonis, cablin patchouli herb, 广蕉香), $85 \mathrm{~g}$; Hong Jing Tian (Herba Rhodiolae, rose-boot, 红景天), 85 g; Yu Xing Cao (Herba Houttuyniae, heartleaf houttuynia herb, 鱼腥草), $255 \mathrm{~g}$; Da Huang (Radix et Rhizoma Rhei, rhubarb root and rhizome, 大黄), $51 \mathrm{~g} ; \mathrm{Ku}$ Xing Ren (Semen Armeniacae Amarum, bitter apricot seed, 苦杏仁) $85 \mathrm{~g}$; and Gan Cao (Radix Glycyrrhizae, liquorice root, 甘草), 85 g [7]. Lianhua Qingwen, which was approved by China Food and Drug Administration (CFDA) in 2004, was the first traditional Chinese medicine that entered the rapid drug approval channel of the CFDA and was used to prevent severe acute respiratory syndrome (SARS). It has been widely used for more than ten years and has become a representative traditional Chinese medicine for respiratory infectious diseases. Pneumonia is one of the most common infections in individuals of all ages, and morbidity of pneumonia increases with age [8]; it is also a significant cause of global mortality. In December 2019, a group of patients with typical pneumonia symptoms of unknown pathogenic factors was described in China, and patients with similar symptoms were quickly detected in other regions. The official name was announced as coronavirus disease 2019 (COVID-19) by the World Health Organization (WHO) on February 11, 2020. This virus has been a major emerging global public health event with no antiviral treatment or vaccine [9]. As of August 6, 2020, the Situation Report-199 of the WHO reported 18,614,177 cases of confirmed COVID-19 pneumonia and 702,642 deaths worldwide [10]. In addition to its impact on health, the COVID-19 pandemic has also led to social, economic, and political damage [11]. The National Health Commission of the People's Republic of China published guidelines for the diagnosis and treatment of COVID-19 pneumonia (Trial version from the Fourth/Fifth/ Sixth/Seventh Edition) and recommended Lianhua Qingwen as a traditional Chinese medicine for COVID-19 pneumonia [12]. Some studies have suggested that Lianhua Qingwen has a therapeutic effect on pneumonia, including COVID-19 pneumonia. However, no consensus has been reached. Therefore, we performed a meta-analysis to investigate and compare the therapeutic effect of Lianhua Qingwen with conventional drugs on pneumonia symptoms.

\section{Materials and Methods}

2.1. Selection Criteria. An electronic search of PubMed, Embase, the Cochrane Library, Foreign Medical Literature Retrieval Service (FRMS), Wanfang (a Chinese database), VIP (a Chinese database), and CNKI (a Chinese database) was performed from the date of inception until July 27, 2020. For the English databases, the following terms were searched in the title, keywords, or abstract: ("Lianhuaqinwen" or "Lianhua Qingwen") and ("Pneumonia" or "Lung Inflammation" or "Pulmonary Inflammation" or "COVID-19" or "Coronavirus disease" or "Novel coronavirus"). For the
Chinese databases, the search terms were "Lianhuaqingwen" and ("Feiyan" or "Feibuyanzheng" or "COVID-19" or "Guanzhuangbindu").

2.2. Study Inclusion and Exclusion Criteria. Studies were selected based on the following criteria: (1) experimental studies; (2) pneumonia was diagnosed according to clinical symptoms, laboratory tests, chest X-ray results, or relevant diagnostic criteria; (3) the Lianhua Qingwen group was treated with Lianhua Qingwen or Lianhua Qingwen combined with conventional drugs (the conventional drug group was given conventional antibiotics, antiviral drugs, or symptomatic treatment); and (4) relevant data of the efficacy index (i.e., flu-like symptoms (fever, cough, sore throat, nausea, diarrhoea, loss of appetite, fatigue, muscle pain, and headache); sputum; pulmonary rales; shortness of breath; breathlessness; chest tightness; pulmonary imaging improvement; curative effect; healing period; conversion of severe cases; and adverse reactions) were provided. The following types of studies were excluded: letters, case reports, those with duplicate or incomplete data (including those in which the efficacy index did not meet the inclusion criteria), reviews or commentaries, animal-based studies, irrelevant studies, and those with a non-Western medicine control.

2.3. Data Extraction and Quality Evaluation. The following information was collected: the first author's name, year of publication, age, sex, sample size, efficacy index, and adverse reactions. All data were extracted directly from the included studies, and no special conversion formula was used. Continuous variables included the total number of patients with symptoms (reported as the mean and standard deviation), and categorical variables included the number of patients with symptoms and the number of patients without symptoms. Disagreements were resolved by a panel discussion with other reviewers. The quality of the eligible studies was evaluated by the CONSORT statement [13] and the Jadad scale [14], which considered sample size determination, randomization, blinding, and loss to follow-up/ withdrawal; scores of $0-2,3-4$, and 5-7 were rated as low, moderate, and high quality, respectively. The Cochrane Collaboration risk of bias tool [15], which consists of seven items, was used to classify each item as low risk of bias, high risk of bias, and unclear risk of bias.

2.4. Statistical Analysis. Statistical analysis was performed using Stata 14 (Stata Corporation, College Station, TX, USA). Continuous variables are expressed as the weighted mean difference (WMD) and 95\% confidence interval (CI), and categorical variables are expressed as the odds ratio (OR) and 95\% CI. Heterogeneity was evaluated with the $I^{2}$ statistic: when $I^{2}>50 \%$, indicating obvious heterogeneity, a random-effects model was used; otherwise, a fixed-effects model was used $[16,17]$. Subgroup, sensitivity, and regression analyses were used to explore possible sources of heterogeneity. Subgroup analysis was performed based on the pneumonia type or flu-like symptoms. Sensitivity 
analysis was performed to assess the influence of each study on a pooled estimate by excluding studies individually and rerunning the meta-analysis. Publication bias was assessed by Begg's test and Egger's test, and visual estimation (via a funnel plot) was also considered; if bias existed, the "trim and fill" method was used to assess publication bias [18]. A $P$ value $<0.05$ was considered statistically significant.

\section{Results}

3.1. Search Results. The detailed flowchart of article selection is presented in Figure 1. A total of 314 citations were identified, and of these, 148 duplicate citations were removed; 59 citations were excluded for irrelevant or animal studies by screening the title and abstract. After full-text screening of the remaining 107 citations, an additional 65 citations were excluded due to the reasons given in Figure 1. Ultimately, 42 experimental Chinese studies involving 3793 subjects were subjected to the meta-analysis. The publication year ranged from 2012 to 2020 [19-60]. Of these studies, 35 researched common pneumonia (including viral pneumonia, mycoplasma pneumonia, community-acquired pneumonia, bronchial pneumonia, bacterial pneumonia, or pneumonia), and the remaining 7 studies researched COVID-19 pneumonia (diagnosed according to the National Health Commission of the People's Republic of China published guidelines for the diagnosis and treatment of COVID-19 pneumonia: Trial version from the Fourth/Fifth Edition). The control groups were given conventional antibiotics, antiviral drugs, or symptomatic treatment. The main characteristics and quality scores of the eligible studies on common pneumonia and COVID-19 pneumonia are shown in Table 1. Regarding the risk of bias assessment, 33 studies reported randomization (of which 12 studies clearly described the randomized methods), and the remaining 9 studies were grouped according to interventions or the hospitalization order. Only one study reported allocation concealment and blinding method, since the symptom indicators needed to be measured by instruments or humans. Therefore, most studies with blinded participants and personnel and a blinded outcome assessment were classified as "unclear risk of bias." Concerning incomplete outcome data and selective reporting, since most studies reported complete data on all symptom indicators, there was a low risk of attrition bias and reporting bias. The percentage of risk of bias is shown in Figure 2.

\subsection{Efficacy Evaluation of Common Pneumonia}

3.2.1. Flu-Like Symptoms. Thirty-one studies compared the duration of flu-like symptoms between the Lianhua Qingwen and conventional drug groups. Among these studies, 29 reported the symptom remission time for fever, and 26 reported the symptom remission time for cough. A short duration of flu-like symptoms was observed in the Lianhua Qingwen group (WMD $=-1.81,95 \% \mathrm{CI}=-2.12$ to -1.50 , $P<0.001)$. Subgroup analysis showed significant differences in the durations of fever (WMD $=-1.58,95 \% \mathrm{CI}=-1.99$ to $-1.17, P<0.001)$ and cough $(\mathrm{WMD}=-2.07,95 \% \mathrm{CI}=-2.57$ to $-1.57, P<0.001)$ between the Lianhua Qingwen and conventional drug groups. Figure 3 shows a forest plot of flulike symptoms for common pneumonia.

\subsubsection{Other Symptoms}

Sputum. Four studies reported the effects of Lianhua Qingwen and those of conventional drugs on the duration of sputum. The duration of sputum was shorter in the Lianhua Qingwen group than in the conventional drug group ( $\mathrm{WMD}=-1.10,95 \% \mathrm{CI}=-1.50$ to -0.70 , $P<0.001)$.

Pulmonary Rale. Eighteen studies reported an effect on the duration of pulmonary rale. The duration of fever was shorter in the Lianhua Qingwen group than in the conventional drug group (WMD $=-2.03,95 \%$ $\mathrm{CI}=-2.74$ to $-1.31, P<0.001)$.

Pulmonary Imaging Improvement. Seven studies reported the duration of pulmonary imaging improvement. The difference between the Lianhua Qingwen group and the conventional drug group was statistically significant $(\mathrm{WMD}=-1.88,95 \% \mathrm{CI}=-2.28$ to -1.47 , $P<0.001)$.

Curative Effect. Thirty studies reported the curative effect of Lianhua Qingwen and that of conventional drugs. The difference between the Lianhua Qingwen group and the conventional drug group was statistically significant $(\mathrm{OR}=3.65,95 \% \mathrm{CI}=2.81$ to $4.76, P<0.001)$.

Healing Period. Twelve studies reported the healing period of the Lianhua Qingwen and conventional drug groups. The Lianhua Qingwen group had a shorter healing period than the conventional drug group $(\mathrm{WMD}=-1.68,95 \% \mathrm{CI}=-2.62$ to $-0.74, P<0.001)$.

Results from the quantitative analysis of common pneumonia are shown in Table 2. Forest plots of other symptoms related to common pneumonia are shown in Figure S1.

\subsection{Efficacy Evaluation of COVID-19 Pneumonia}

3.3.1. Flu-Like Symptoms. Four studies compared improving flu-like symptoms (fever, cough, sore throat, nausea, diarrhoea, loss of appetite, fatigue, muscle pain, and headache) between the Lianhua Qingwen and conventional drug groups. The difference in improving flu-like symptoms between the Lianhua Qingwen and conventional drug groups was statistically significant $(\mathrm{OR}=3.18,95 \% \mathrm{CI}=2.36$ to $4.29, P<0.001)$. Subgroup analysis indicated significant associations with fever $(\mathrm{OR}=4.05,95 \% \mathrm{CI}=1.78$ to 6.59 , $P<0.001)$, cough $(\mathrm{OR}=3.43,95 \% \mathrm{CI}=1.87$ to 6.29 , $P<0.001)$, fatigue $(\mathrm{OR}=2.82,95 \% \mathrm{CI}=1.44$ to 5.53 , $P=0.003)$, and muscle pain $(\mathrm{OR}=5.01,95 \% \mathrm{CI}=1.44$ to $17.40 P=0.011)$. However, subgroup analysis revealed nonsignificant associations with sore throat $(\mathrm{OR}=0.31,95 \%$ $\mathrm{CI}=0.03$ to $2.99, \quad P=0.314)$, nausea $(\mathrm{OR}=1.98,95 \%$ $\mathrm{CI}=0.49$ to $7.95, P=0.336)$, diarrhoea $(\mathrm{OR}=1.28,95 \%$ $\mathrm{CI}=0.18$ to $9.30, P=0.810)$, loss of appetite $(\mathrm{OR}=6.05,95 \%$ 


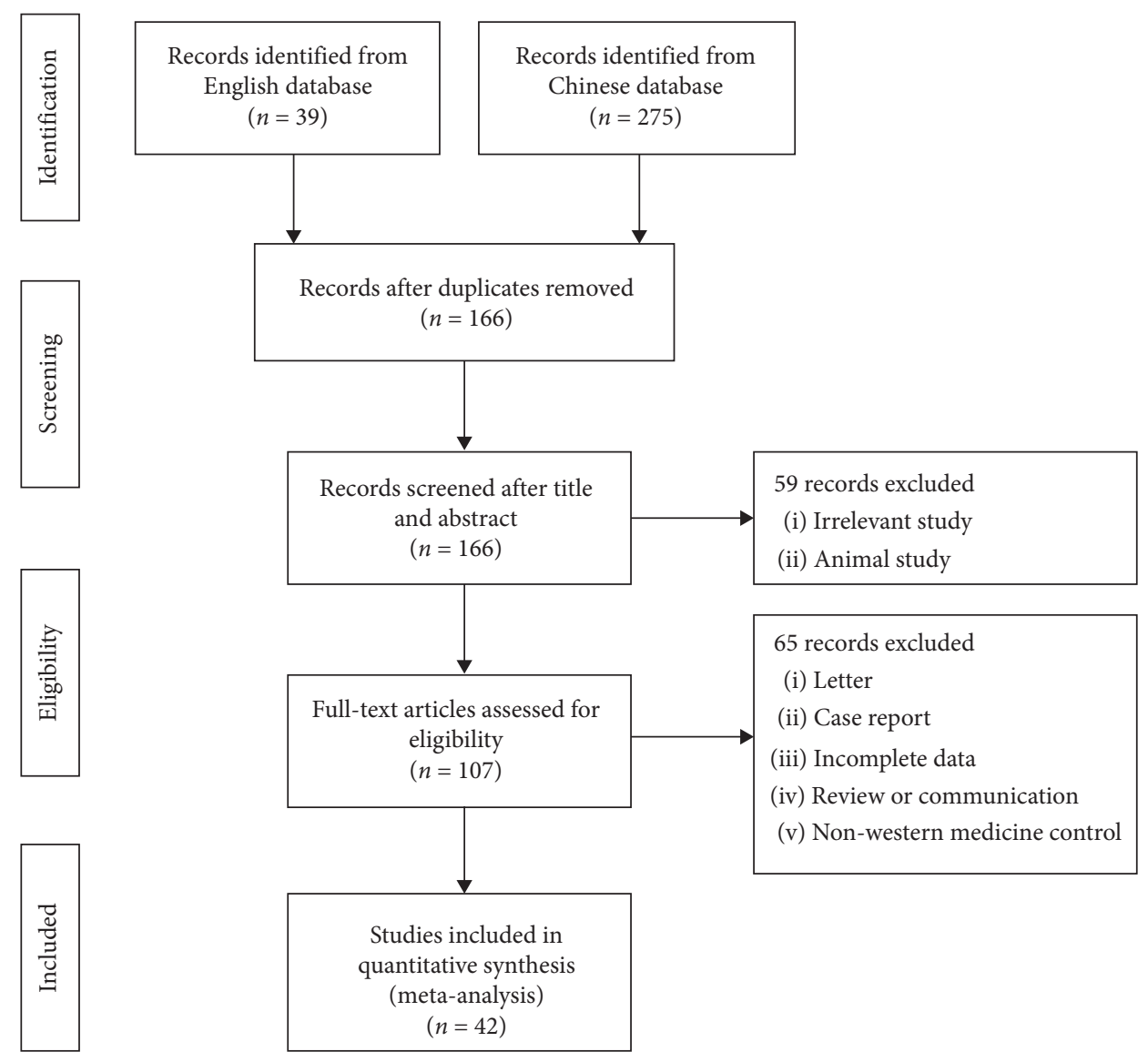

FIgURE 1: Flowchart of the search and selection process.

$\mathrm{CI}=0.69$ to $53.14, P=0.104)$, and headache $(\mathrm{OR}=2.67,95 \%$ $\mathrm{CI}=0.30$ to $23.83, P=0.378$ ). Figure 3 shows a forest plot of flu-like symptoms for COVID-19 pneumonia.

\subsubsection{Other Symptoms}

Sputum. Three studies reported the improvement in sputum. The difference in sputum improvement was statistically significant between the Lianhua Qingwen and conventional drug groups $(\mathrm{OR}=4.30,95 \%$ $\mathrm{CI}=1.01$ to $18.22, P=0.048)$.

Shortness of Breath. Three studies compared the effect of Lianhua Qingwen with that of conventional drugs on the shortness of breath, and Lianhua Qingwen was associated with a better effect than conventional drugs $(\mathrm{OR}=10.62,95 \% \mathrm{CI}=3.71$ to $30.40, P<0.001)$.

Breathlessness. Three studies reported improvement in breathlessness in the Lianhua Qingwen and conventional drug groups. However, it failed to identify a statistically significant difference between the two groups $(\mathrm{OR}=4.81,95 \% \mathrm{CI}=0.97$ to $23.86, P=0.055)$.

Chest Tightness. Three studies reported the curative effect of chest tightness. A statistically significant difference was identified between the Lianhua Qingwen group and the conventional drug group $(\mathrm{OR}=3.02$, $95 \% \mathrm{CI}=1.23$ to $7.42, P=0.016$ ).
Pulmonary Imaging Improvement. Five studies reported improvement in pulmonary imaging. A statistically significant difference was identified between the Lianhua Qingwen group and the conventional drug group $(\mathrm{OR}=1.77,95 \% \mathrm{CI}=1.29$ to $2.42, P<0.001)$.

Curative Effect. Five studies compared the curative effect of Lianhua Qingwen with that of conventional drugs. A statistically significant difference was identified between the two groups $(\mathrm{OR}=2.49,95 \% \mathrm{CI}=1.76$ to $3.53, P<0.001)$.

Healing Period. Two studies reported the healing period of the Lianhua Qingwen and conventional drug groups. The Lianhua Qingwen group was associated with a shorter healing period than the conventional drug group (WMD $=-2.06,95 \% \mathrm{CI}=-3.36$ to -0.75 , $P=0.002)$.

Conversion of Severe Cases. Four studies reported the conversion of severe cases in both groups. A statistically significant difference was detected between the two groups $(\mathrm{OR}=0.46,95 \% \mathrm{CI}=0.27$ to 0.77 , $P=0.003)$.

Results from the quantitative analysis of COVID-19 pneumonia are shown in Table 2. Forest plots of other symptoms for COVID-19 pneumonia are shown in Figure S2. 


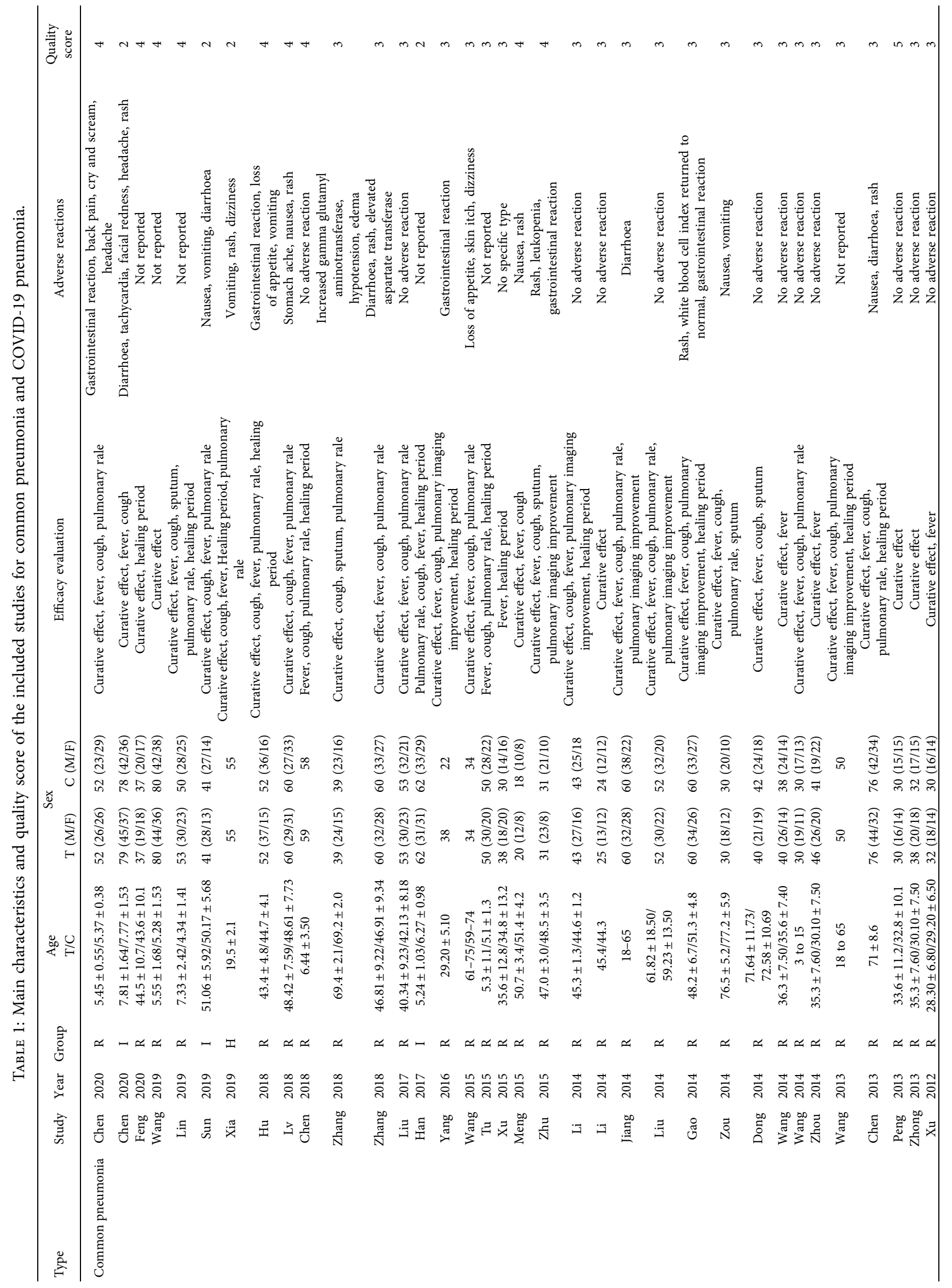




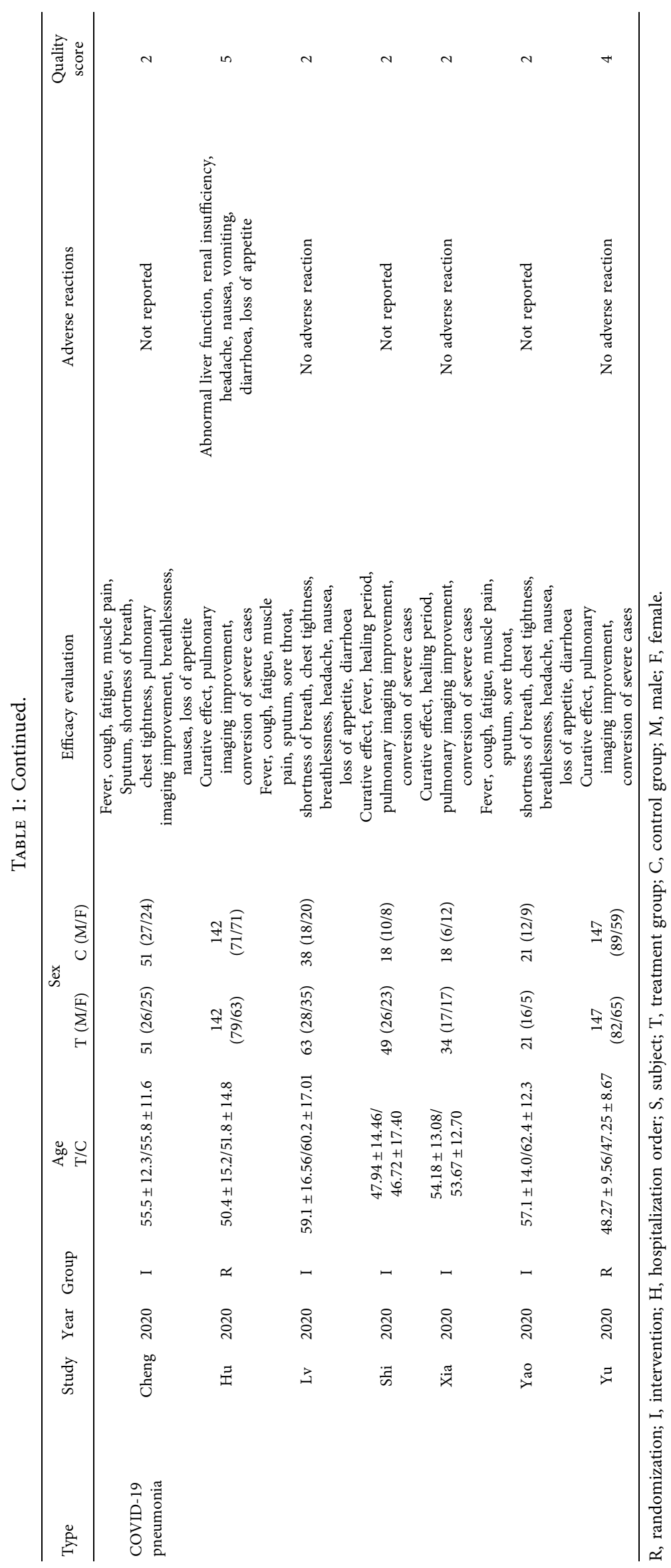




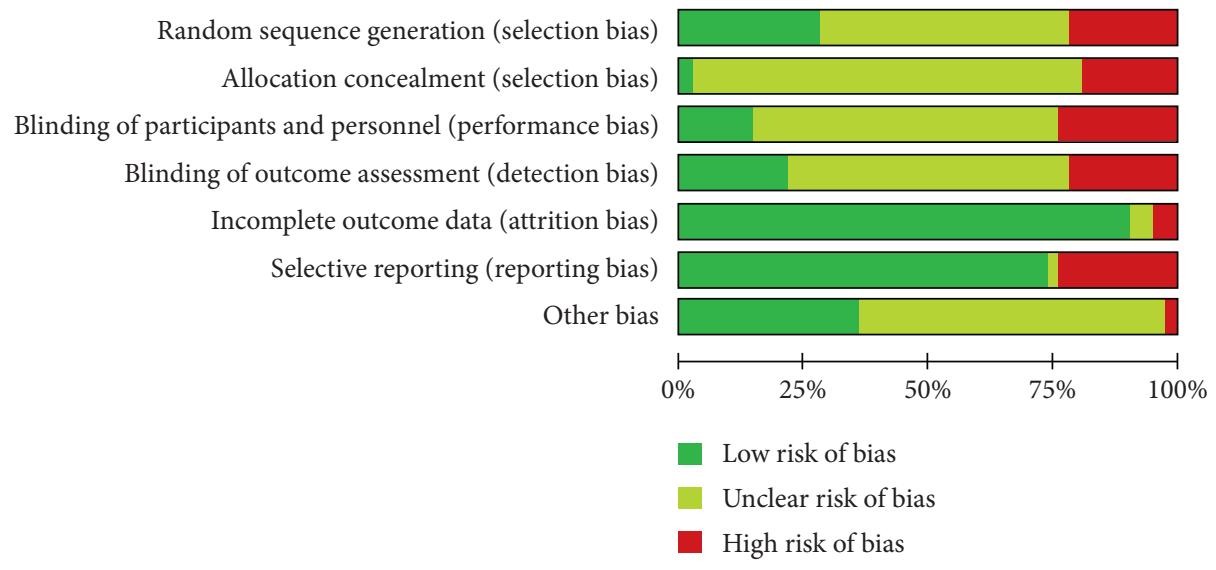

Figure 2: Percentage of risk of bias for all qualified studies.

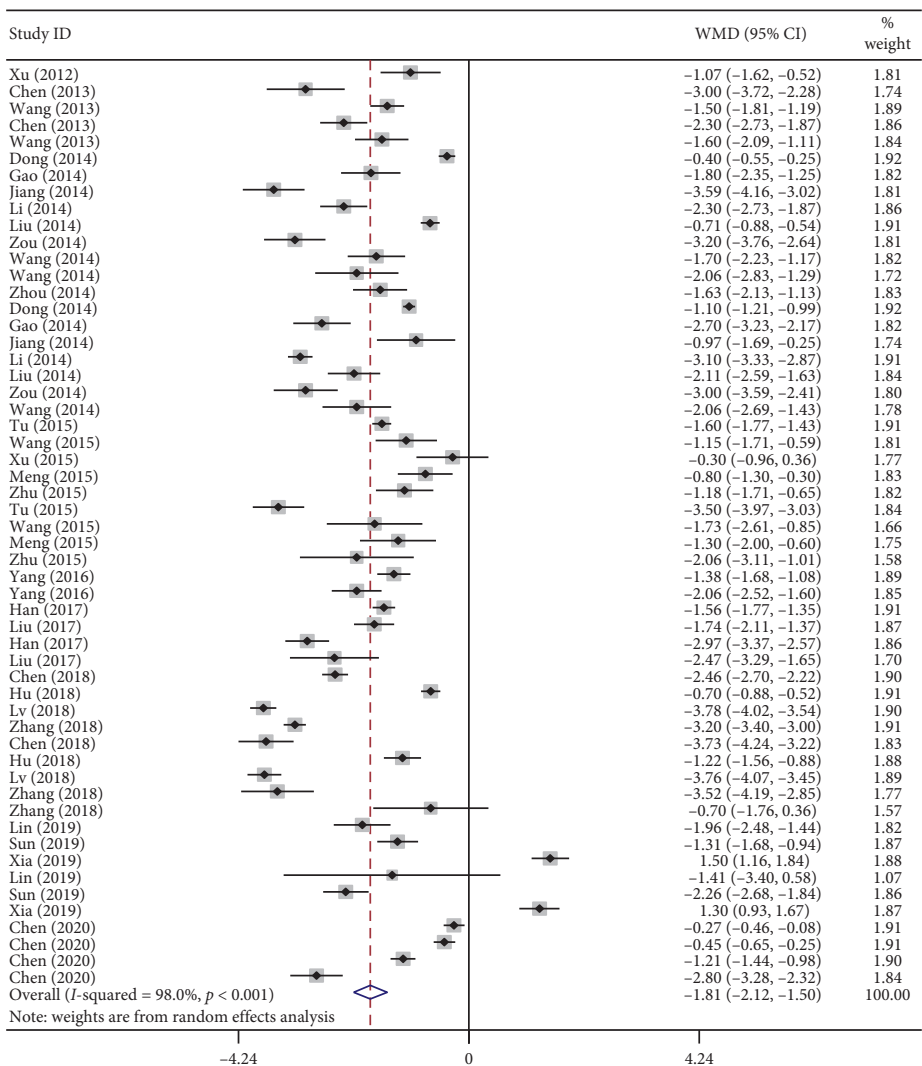

(a)

Figure 3: Continued. 


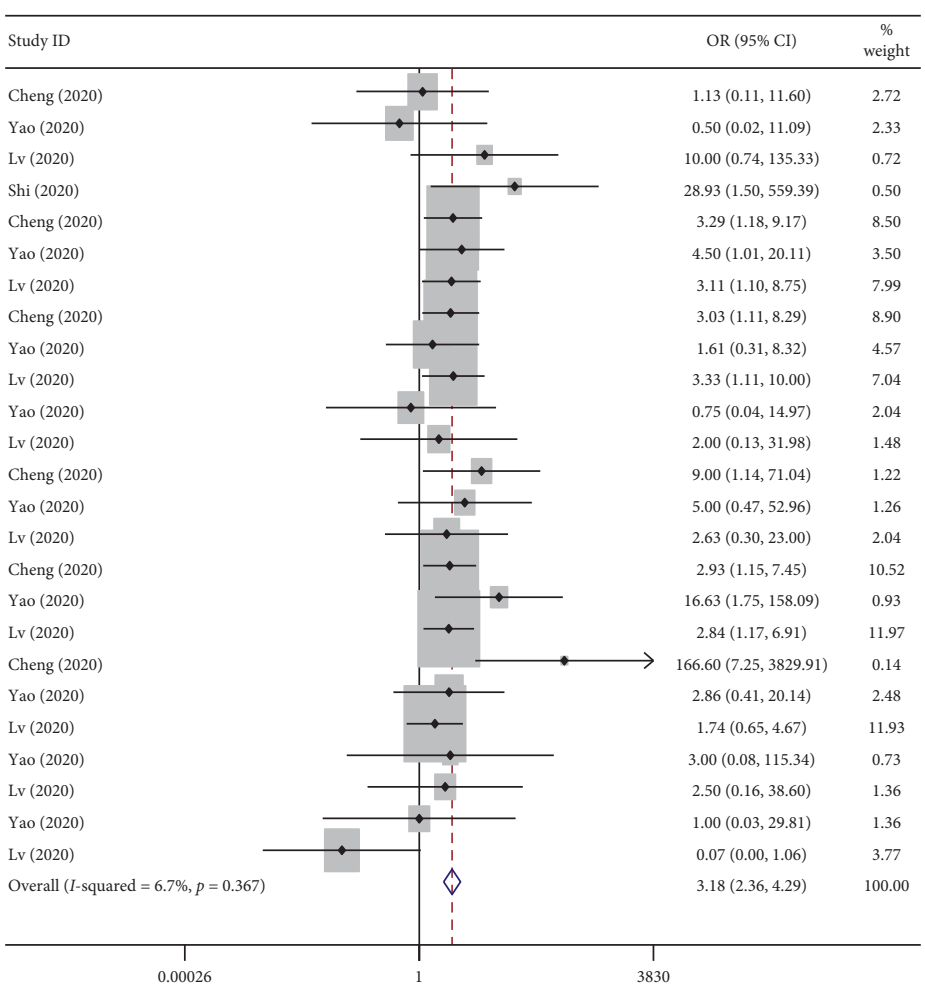

(b)

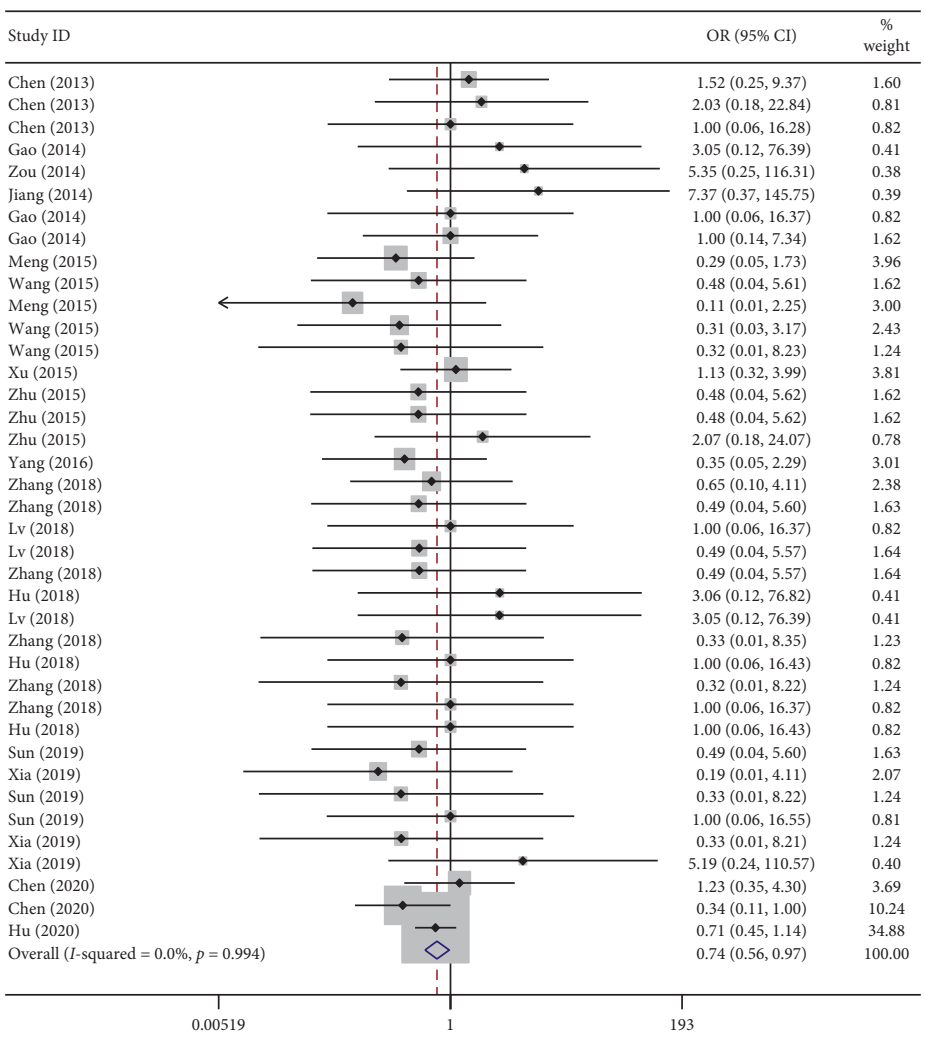

(c)

FIGURE 3: Forest plot of the comparison between the Lianhua Qingwen group and the conventional drug group on flu-like symptoms and adverse reactions: (a) flu-like symptoms for common pneumonia; (b) flu-like symptoms for COVID-19 pneumonia; (c) adverse reactions. 
TABLE 2: The quantitative analysis of common pneumonia and COVID-19 pneumonia.

\begin{tabular}{|c|c|c|c|c|c|c|c|}
\hline \multirow{2}{*}{ Subject } & \multirow{2}{*}{ Efficacy index } & \multirow{2}{*}{ Effect value } & \multirow[b]{2}{*}{$95 \% \mathrm{CI}$} & \multirow{2}{*}{$P_{\text {Effect value }}$} & \multicolumn{2}{|c|}{ Heterogeneity } & \multirow{2}{*}{$M$} \\
\hline & & & & & $I^{2}$ & $P$ & \\
\hline \multirow{8}{*}{ Common pneumonia } & Flu-like symptoms & $\mathrm{WMD}=-1.81$ & $-2.12,-1.50$ & $<0.001$ & 98.0 & $<0.001$ & $\mathrm{R}$ \\
\hline & Fever & $\mathrm{WMD}=-1.58$ & $-1.99,-1.17$ & $<0.001$ & 98.3 & $<0.001$ & $\mathrm{R}$ \\
\hline & Cough & $\mathrm{WMD}=-2.07$ & $-2.57,-1.57$ & $<0.001$ & 97.7 & $<0.001$ & $\mathrm{R}$ \\
\hline & Sputum & $\mathrm{WMD}=-1.10$ & $-1.50,-0.70$ & $<0.001$ & 62.2 & 0.047 & $\mathrm{R}$ \\
\hline & Pulmonary rales & $\mathrm{WMD}=-2.03$ & $-2.74,-1.31$ & $<0.001$ & 97.1 & $<0.001$ & $\mathrm{R}$ \\
\hline & Pulmonary imaging improvement & $\mathrm{WMD}=-1.88$ & $-2.28,-1.47$ & $<0.001$ & 56.5 & 0.032 & $\mathrm{R}$ \\
\hline & Curative effect & $\mathrm{OR}=3.65$ & $2.81,4.76$ & $<0.001$ & 0.0 & 0.945 & $\mathrm{~F}$ \\
\hline & Healing period & $\mathrm{WMD}=-1.68$ & $-2.62,-0.74$ & $<0.001$ & 97.0 & $<0.001$ & $\mathrm{R}$ \\
\hline \multirow{18}{*}{ COVID-19 pneumonia } & Flu-like symptoms & $\mathrm{OR}=3.18$ & $2.36,4.29$ & $<0.001$ & 6.7 & 0.367 & $\mathrm{~F}$ \\
\hline & Fever & $\mathrm{OR}=4.05$ & $1.78,6.59$ & $<0.001$ & 0.0 & 0.548 & $\mathrm{~F}$ \\
\hline & Cough & $\mathrm{OR}=3.43$ & $1.87,6.29$ & $<0.001$ & 7.7 & 0.338 & $\mathrm{~F}$ \\
\hline & Sore throat & $\mathrm{OR}=0.31$ & $0.03,2.99$ & 0.314 & 39.8 & 0.197 & $\mathrm{~F}$ \\
\hline & Nausea & $\mathrm{OR}=1.98$ & $0.49,7.95$ & 0.336 & 7.4 & 0.340 & $\mathrm{~F}$ \\
\hline & Diarrhoea & $\mathrm{OR}=1.28$ & $0.18,9.30$ & 0.810 & 0.0 & 0.638 & $\mathrm{~F}$ \\
\hline & Loss of appetite & $\mathrm{OR}=6.05$ & $0.69,53.14$ & 0.104 & 74.5 & 0.020 & $\mathrm{R}$ \\
\hline & Fatigue & $\mathrm{OR}=2.82$ & $1.44,5.53$ & 0.003 & 0.0 & 0.757 & $\mathrm{~F}$ \\
\hline & Muscle pain & $\mathrm{OR}=5.01$ & $1.44,17.40$ & 0.011 & 0.0 & 0.723 & $\mathrm{~F}$ \\
\hline & Headache & $\mathrm{OR}=2.67$ & $0.30,23.83$ & 0.378 & 0.0 & 0.938 & $\mathrm{~F}$ \\
\hline & Sputum & $\mathrm{OR}=4.30$ & $1.01,18.22$ & 0.048 & 61.9 & 0.073 & $\mathrm{R}$ \\
\hline & Shortness of breath & $\mathrm{OR}=10.62$ & $3.71,30.40$ & $<0.001$ & 0.0 & 0.751 & $\mathrm{~F}$ \\
\hline & Breathlessness & $\mathrm{OR}=4.81$ & $0.97,23.86$ & 0.055 & 0.0 & 0.672 & $\mathrm{~F}$ \\
\hline & Chest tightness & $\mathrm{OR}=3.02$ & $1.23,7.42$ & 0.016 & 31.5 & 0.232 & $\mathrm{~F}$ \\
\hline & Pulmonary imaging improvement & $\mathrm{OR}=1.77$ & $1.29,2.42$ & $<0.001$ & 43.7 & 0.130 & $\mathrm{~F}$ \\
\hline & Curative effect & $\mathrm{OR}=2.49$ & $1.76-3.53$ & $<0.001$ & 29.8 & 0.234 & $\mathrm{~F}$ \\
\hline & Healing period & $\mathrm{WMD}=-2.06$ & $-3.36,0.75$ & 0.002 & 0.0 & 0.810 & $\mathrm{~F}$ \\
\hline & Conversion of severe cases & $\mathrm{OR}=0.46$ & $0.27,0.77$ & 0.003 & 18.2 & 0.295 & $\mathrm{~F}$ \\
\hline \multirow{2}{*}{ All } & Adverse reactions & $\mathrm{OR}=0.74$ & $0.56,0.97$ & 0.029 & 0.0 & 0.994 & $\mathrm{~F}$ \\
\hline & Common pneumonia & $\mathrm{OR}=0.75$ & $0.54,1.05$ & 0.097 & 0.0 & 0.992 & $\mathrm{~F}$ \\
\hline
\end{tabular}

M, model; R, random-effects model; F, fixed-effects model.

3.4. Adverse Reactions. Eighteen studies (17 for common pneumonia and one for COVID-10 pneumonia) reported adverse reactions, including diarrhoea, rash, gastrointestinal reaction, headache, nausea, vomiting, abnormal liver function, and renal insufficiency, of the Lianhua Qingwen and conventional drugs groups. A significant statistical difference was detected between the two groups $(\mathrm{OR}=0.74$, $95 \% \mathrm{CI}=0.56$ to $0.97, P=0.029)$. Subgroup analysis revealed no significant difference regarding common pneumonia $(\mathrm{OR}=0.75,95 \% \mathrm{CI}=0.54$ to $1.05, P=0.097)$. Since only one study of COVID-19 pneumonia reported adverse reactions, no subgroup analysis was performed. Results from the quantitative analysis of adverse reactions are shown in Table 2. Figure 3 shows a forest plot of adverse reactions.

3.5. Sensitivity Analysis and Meta-Regression. Sensitivity analysis was performed by deleting individual studies to assess the stability of the results. For common pneumonia, there was no significant alteration in any efficacy evaluation index. For COVID-19 pneumonia, changes in the following pooled results were observed: improvement in sputum ( $\mathrm{WMD}=4.30,95 \% \mathrm{CI}=0.36$ to 47.40$)$, chest tightness $(\mathrm{OR}=3.02,95 \% \mathrm{CI}=0.77$ to 28.11$)$, and pulmonary imaging improvement $(\mathrm{OR}=1.77,95 \% \mathrm{CI}=0.95$ to 3.41$)$. Sensitivity analysis revealed changes in adverse reactions $(\mathrm{OR}=0.74$,
$95 \% \mathrm{CI}=0.54$ to 1.05$)$. The sensitivity analysis results are shown in Table 3.

For common pneumonia, high heterogeneity was found in the following indexes: flu-like symptoms $\left(I^{2}=98.0\right)$, sputum $\left(I^{2}=62.2\right)$, pulmonary rales $\left(I^{2}=97.1\right)$, pulmonary imaging improvement $\left(I^{2}=56.5\right)$, and healing period $\left(I^{2}=97.0\right)$. However, subgroup analysis did not indicate heterogeneity, and no source of heterogeneity was found in the sensitivity analysis. Therefore, a meta-regression was performed to explore the likely source of heterogeneity, such as publication year, sample size, and quality score. The results indicated that these covariates could not explain all the heterogeneity and that the heterogeneity might be related to the inconsistency of conventional drugs used in each study and the individual absorption of drugs. For common pneumonia, high heterogeneity was found in the sputum index. Since there are limited studies on COVID-19 pneumonia, meta-regression was not performed. The results of the meta-regression are summarized in Table 4.

3.6. Publication Bias. The index involving the largest number of qualified studies was used to assess publication bias. For common pneumonia, 30 studies that reported curative effects were used to assess publication bias, and neither Begg's test nor Egger's test found any visible evidence for publication bias $\left(P_{\text {Begg }}=0.153, P_{\text {Egger }}=0.577\right)$. Since 
TABLE 3: Sensitivity analysis of common pneumonia and COVID-19 pneumonia.

\begin{tabular}{|c|c|c|c|}
\hline Subject & Efficacy index & Effect value & $95 \% \mathrm{CI}$ \\
\hline \multirow{7}{*}{ Common pneumonia } & Flu-like symptoms & $\mathrm{WMD}=-1.81$ & $-2.17,-1.47$ \\
\hline & Sputum & $\mathrm{WMD}=-1.10$ & $-1.94,-0.52$ \\
\hline & Pulmonary rales & $\mathrm{WMD}=-2.03$ & $-2.87,-1.21$ \\
\hline & Pulmonary imaging improvement & $\mathrm{WMD}=-1.88$ & $-2.45,-1.37$ \\
\hline & Curative effect & $\mathrm{OR}=3.65$ & $2.69,5.13$ \\
\hline & Healing period & WMD-1.68 & $-2.80,-0.53$ \\
\hline & Adverse reaction & $\mathrm{OR}=0.75$ & $0.50,1.19$ \\
\hline \multirow{9}{*}{ COVID1-19 pneumonia } & Flu-like symptoms & $\mathrm{OR}=3.18$ & $2.18,4.63$ \\
\hline & Sputum* & $\mathrm{OR}=4.30$ & $0.36,47.40$ \\
\hline & Shortness of breath & $\mathrm{OR}=10.62$ & $2.80,66.79$ \\
\hline & Breathlessness & $\mathrm{OR}=4.81$ & 0.29 .42 .01 \\
\hline & Chest tightness* & $\mathrm{OR}=3.02$ & $0.77,28.11$ \\
\hline & Pulmonary imaging improvement* & $\mathrm{OR}=1.77$ & $0.95,3.41$ \\
\hline & Curative effect & $\mathrm{OR}=2.49$ & $1.58,4.85$ \\
\hline & Healing period & $\mathrm{WMD}=-2.06$ & $-4.01,0.01$ \\
\hline & Conversion of severe cases & $\mathrm{OR}=0.46$ & $0.10,0.92$ \\
\hline All & Adverse reactions* & $\mathrm{OR}=0.74$ & $0.54,1.05$ \\
\hline
\end{tabular}

*The sensitivity analysis results indicated that deleting individual studies would change the combined results of the meta-analysis.

TABLE 4: Results of the meta-regression.

\begin{tabular}{|c|c|c|c|c|}
\hline Subject & & Publication year & Sample size & Quality score \\
\hline \multirow{5}{*}{ Common pneumonia } & Flu-like symptoms & $P=0.190$ & $P=0.070$ & $P=0.330$ \\
\hline & Sputum & $P=0.947$ & $P=0.823$ & $P=0.131$ \\
\hline & Pulmonary rales & $P=0.150$ & $P=0.480$ & $P=0.510$ \\
\hline & Pulmonary imaging improvement & $P=1.000$ & $P=0.770$ & $P=0.960$ \\
\hline & Healing period & $P=0.850$ & $P=0.990$ & $P=0.150$ \\
\hline
\end{tabular}

there were limited studies on curative effects for COVID-19 pneumonia, there was no assessment of publication bias for COVID-19 pneumonia. Concerning adverse reactions, neither Begg's test nor Egger's test found any visible evidence for publication bias $\left(P_{\text {Begg }}=0.904, P_{\text {Egger }}=0.512\right)$. Symmetrical funnel plots are shown in Figure 4.

\section{Discussion}

Antibiotics are a traditional choice used to treat treating pneumonia; however, the inappropriate use of antibiotics leads to increasing problems such as antimicrobial resistance and is related to multiple adverse effects $[61,62]$. According to recent research, the death toll will reach 10 million by 2050 , due to infections caused by resistant bacteria [63]. A simple infection may be fatal in the future, and it is of crucial importance to identify and find an effective and safe alternative for treating pneumonia. Traditional Chinese medicine has been extensively used, as it offers unique advantages in regulating homeostasis and improving immunity, prevention, and control. Lianhua Qingwen is a traditional Chinese medicine that can relieve fever, act as an antitussive expectorant, dispel a cold, eliminate inflammation, provide endogenous heat, act as an antiviral agent, and improve immunity [64-66]. Some studies have reported that Lianhua Qingwen is effective against viral or bacterial infections in pneumonia $[40,46,48,67]$; however, its principle of treatment remains inconclusive. Animal experiments have shown that Lianhua Qingwen plays a protective role in lung tissue damage by inhibiting inflammatory cell infiltration and improving protein expression in alveolar epithelial and pulmonary vascular endothelial cells [68]. Pharmacological experiments have indicated that a high dosage of Lianhua Qingwen alters the effectiveness of cyclooxygenase- 2 in the arachidonic acid metabolic pathway and decreases decrease virus replication, producing significant improvements in lung inflammation [69]. It was recently reported that Lianhua Qingwen plays a significant role in the treatment of pneumonia by significantly reducing pathological changes, which might be correlated with the levels of enzymes regulated by Lianhua Qingwen, such as glutathione peroxidase, lactate dehydrogenase, superoxide dismutase, and malonaldehyde [65]. Previous studies reviewed historical records and prevention measures against SARS, H1N1 influenza, and Middle East respiratory syndrome (MERS) and reported that traditional Chinese medicine might be an alternative for the prevention and treatment of COVID-19 pneumonia [70-73]. Lu et al. [74] reported that Lianhua Qingwen plays an active role in controlling COVID-19 pneumonia, especially mild symptoms.

Our meta-analysis was performed to compare the effect of Lianhua Qingwen and conventional drugs on pneumonia symptoms. For common pneumonia, this study observed that the Lianhua Qingwen group was associated with shorter durations of flu-like symptoms, sputum, pulmonary rale, pulmonary imaging improvement, and healing period than 


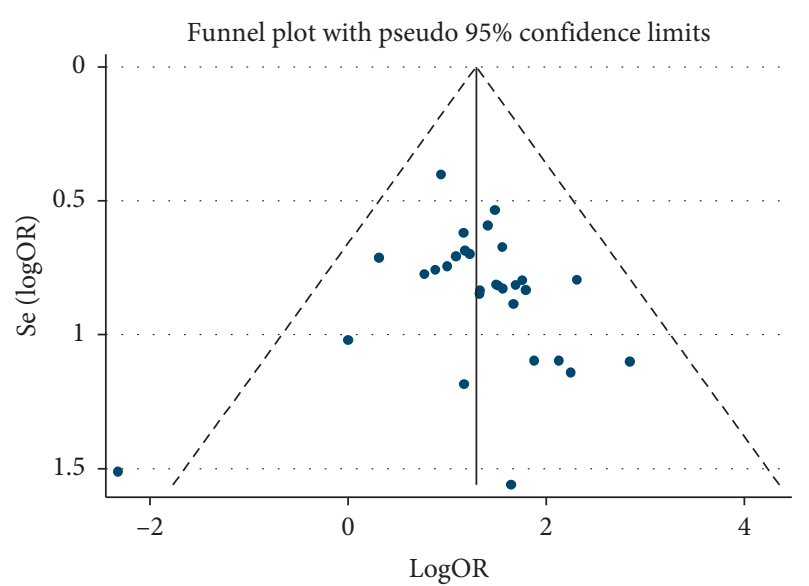

(a)

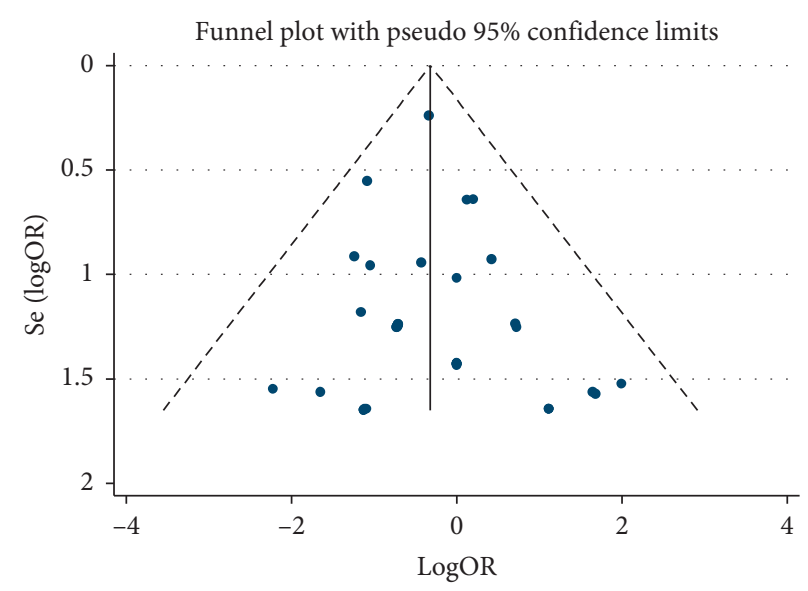

(b)

FIGURE 4: Funnel plots used to assess publication bias: (a) the index of curative effect for common pneumonia; (b) adverse reactions.

conventional drugs; a statistically significant curative effect was also identified. The subgroup analysis based on flu-like symptoms showed statistically significant differences in fever and cough; sensitivity analysis showed that the pooled results related to common pneumonia were stable. A metaanalysis by Zhou et al. [75] revealed that Lianhua Qingwen combined with another treatment for patients with community-acquired pneumonia could improve the effective rate and pulmonary imaging and shorten durations of fever and cough, consistent with our meta-analysis. However, our meta-analysis was more comprehensive, as more new studies were included, and other important indicators of pneumonia, such as pulmonary rale, sputum, and healing period, were also taken into account. For COVID-19 pneumonia, we found that Lianhua Qingwen was more useful for improving flu-like symptoms, sputum, shortness of breath, chest tightness, pulmonary imaging improvement, curative effect, healing period, and conversion of severe cases than conventional drugs. Subgroup analysis indicated statistically significant improvements in fever, cough, fatigue, and muscle pain; however, sensitivity analysis revealed unstable results related to sputum, chest tightness, and pulmonary imaging improvement. This inconsistency might be due to the limited number of eligible studies. Only three qualified studies that reported curative effects of typical clinical symptoms were identified, and a further stratified analysis was unavailable for the meta-analysis. Several recent studies support our conclusion that Lianhua Qingwen has a positive effect on the recovery of common pneumonia and COVID19 pneumonia. Runfeng et al. [67] discovered changes in cytokine profiles, suggesting that Lianhua Qingwen exerts an inhibitory effect on cytokine storms induced by COVID-19 pneumonia and demonstrating that Lianhua Qingwen acts as an anti-coronavirus agent by reducing cytokine release from host cells and inhibiting virus replication. Liu et al. [76] researched the efficacy and safety of integrated Chinese and Western medicines in the treatment of COVID-19 pneumonia and showed that Lianhua Qingwen improved the total effective rate $(\mathrm{RR}=1.26,95 \% \mathrm{CI}=1.01$ to 1.56 ,
$P=0.037)$, fever disappearance rate $(\mathrm{RR}=1.4195 \% \mathrm{CI}=1.21$ to $1.78, P=0.003)$, fatigue disappearance rate $(\mathrm{RR}=1.69$, $95 \% \mathrm{CI}=1.05$ to $2.72, P=0.032$ ), muscle pain disappearance rate $(\mathrm{RR}=2.91,95 \% \mathrm{CI}=1.14$ to $7.38, P=0.025)$, and sputum disappearance rate $(\mathrm{RR}=4.17,95 \% \mathrm{CI}=1.59$ to 10.89 , $P=0.004)$. However, no other analysis was performed to detect possible sources of heterogeneity and assess the stability of the results. Even though the efficacy and outcome indicators were not as complete as ours, the results support our results to a certain extent.

Our study found a statistically significant difference in adverse reactions between the Lianhua Qingwen group and the conventional drug group. Although sensitivity analysis revealed changes in adverse reactions, subgroup analysis showed no significant difference concerning common pneumonia. Yiling Pharmaceutical, which is responsible for research on the production of Lianhua Qingwen, has reported no genotoxicity or nephrotoxicity of Lianhua Qingwen according to systemic toxicology studies [77]. A meta-analysis focused solely on the safety of Lianhua Qingwen that involved 40 RCTs reported that the incidence of adverse reactions in the Lianhua Qingwen group was significantly lower than that in the control group $(\mathrm{RR}=0.562,95 \% \mathrm{CI}=0.412-0.767, P$ value not reported $)$. Subgroup analysis showed that the incidence of digestive system adverse reactions in the Lianhua Qingwen group was also lower than that in the control group $(\mathrm{RR}=0.70,95 \%$ $\mathrm{CI}=0.50$ to $0.97, P$ value not reported), suggesting the safety of Lianhua Qingwen [78]. Previous studies have also shown that Lianhua Qingwen has therapeutic effects on influenza. Three meta-analyses explored the efficacy/safety of Lianhua Qingwen and conventional drugs for treating influenza. Among them, Wang et al. [79] showed that Lianhua Qingwen has a superior curative effect on the treatment of viral influenza, but the incidence of adverse reactions was higher than that of conventional drugs; therefore, clinicians should pay should pay close attention to the safety of Lianhua Qingwen. Niu et al. [80] reported that Lianhua Qingwen was highly safe and had a better curative effect on 
the treatment of influenza than oseltamivir, ribavirin, ammonia yellow capsules, and even Huaqing Pesti capsules. Zhao et al. [81] concluded that Lianhua Qingwen was better than oseltamivir in improving the symptoms of influenza $\mathrm{A}$ virus infection, and no significant drug-related adverse reactions were observed in any of the included studies. Thus, these findings suggest that Lianhua Qingwen exerts better therapeutic effects by improving disease symptoms than conventional drugs, but the safety of Lianhua Qingwen cannot be ignored. Additional studies with a focus on the safety and association of Lianhua Qingwen with diseases should be performed.

Several limitations to our meta-analysis should be noted. First, the quality of the included studies was generally moderate, and only seven studies on COVID-19 pneumonia were included; thus, more high-quality research is needed. Second, the results of some indicators of COVID-19 pneumonia and adverse reactions were unstable. Since the number of studies on COVID-19 pneumonia were limited, publication bias and meta-regression analysis have not been performed. Finally, although English and Chinese articles were retrieved, only Chinese studies were qualified; therefore, language bias might not be excluded.

Some advantages should be mentioned. First, to our knowledge, this is the most comprehensive meta-analysis of the efficacy of Lianhua Qingwen for the treatment of common pneumonia and COVID-19 pneumonia. Second, to our knowledge, this is the first meta-analysis of the use of Lianhua Qingwen for the treatment of pneumonia based on several indicators (i.e., sputum, curative effect, and healing period for common pneumonia; flu-like symptoms, shortness of breath, breathlessness, chest tightness, pulmonary imaging improvement, healing period, and conversion of severe cases for COVID-19 pneumonia). Finally, we determined that Lianhua Qingwen combined with conventional drugs can better promote the recovery of certain clinical symptoms related to common pneumonia and COVID-19 pneumonia than conventional drugs.

\section{Conclusion}

Lianhua Qingwen combined with conventional drugs may be a promising therapy for treating pneumonia, including common pneumonia and COVID-19 pneumonia, but more high-quality studies are needed to explore the efficacy and mechanism of Lianhua Qingwen in treating pneumonia.

\section{Data Availability}

The data used to support the findings of this study are available from the corresponding author upon request.

\section{Conflicts of Interest}

The authors declare that they have no conflicts of interest.

\section{Authors' Contributions}

Caiyun $\mathrm{Hu}$ designed the study. Caiyun $\mathrm{Hu}$ and Mingming Liang retrieved electronic records. Fengfeng Gong and Bin
He performed data extraction and analysis. Dongdong Zhao performed quality evaluation. Caiyun $\mathrm{Hu}$ and Mingming Liang wrote the draft. Guoliang Zhang performed final review and acquired funding.

\section{Acknowledgments}

This study was supported by the COVID-19 Pneumonia of Traditional Chinese Medicine Emergency Project, State Administration of Traditional Chinese Medicine of the People's Republic of China (2020ZYLCYJ02-1).

\section{Supplementary Materials}

Figure S1: forest plot of the comparison between the Lianhua Qingwen group and the conventional drug group on other symptoms related to common pneumonia. Figure S2: forest plot of the comparison between the Lianhua Qingwen group and the conventional drug group on other symptoms related to COVID-19 pneumonia. (Supplementary Materials)

\section{References}

[1] C.-H. Hsu, K.-C. Hwang, C.-L. Chao et al., "The lesson of supplementary treatment with Chinese medicine on severe laboratory-confirmed SARS patients," The American Journal of Chinese Medicine, vol. 34, no. 6, pp. 927-935, 2006.

[2] J. T. F. Lau, P. C. Leung, E. L. Y. Wong et al., "The use of an herbal formula by hospital care workers during the severe acute respiratory syndrome epidemic in Hong Kong to prevent severe acute respiratory syndrome transmission, relieve influenza-related symptoms, and improve quality of life: a prospective cohort study," The Journal of Alternative and Complementary Medicine, vol. 11, no. 1, pp. 49-55, 2005.

[3] P. Zhao, H.-z. Yang, J.-f. Li et al., "A case of autoimmune hepatitis and primary biliary cirrhosis overlap syndrome treated with Chinese herbs," Chinese Journal of Integrative Medicine, vol. 19, no. 6, pp. 468-470, 2013.

[4] K. Peltzer, "Utilization and practice of traditional/complementary/alternative medicine (TM/CAM) in South Africa," African Journal of Traditional, Complementary, and Alternative Medicines: AJTCAM, vol. 6, no. 2, pp. 175-185, 2009.

[5] S. Wu, K. B. Patel, L. J. Booth et al., "Protective essential oil attenuates influenza virus infection: an in vitro study in MDCK cells," BMC Complementary and Alternative Medicine, vol. 10, p. 69, 2010.

[6] C. Y. Liu, X. Q. Li, and S. Q. Cai, "Advances in pharmacology and clinical research of lianhua Qingwen capsules," Pharmacology and Clinics of Chinese Materia Medica, vol. 26, no. 6, pp. 84-85, 2010.

[7] Medical Encyclopedia, "Lianhua Qingwen," 2020, https:// www.wiki8.com/lianhuaqingwenpian_143047/.

[8] S. Jain, D. J. Williams, S. R. Arnold et al., "Community-acquired pneumonia requiring hospitalization among U.S. children," New England Journal of Medicine, vol. 372, no. 9, pp. 835-845, 2015.

[9] N. Zhu, D. Zhang, W. Wang et al., "A novel coronavirus from patients with pneumonia in China, 2019," The New England Journal of Medicine, vol. 382, no. 8, pp. 727-733, 2020.

[10] World Health Organization, Coronavirus Disease 2019 (COVID-19) Situation Report-199, https://www.who.int/docs/ default-source/coronaviruse/situation-reports/20200806covid-19 sitrep-199.pdf?sfvrsn=6b9d262d_2, World Health 
Organization, Geneva, Switzerland, 2020, https://www.who. int/docs/default-source/coronaviruse/situation-reports/ 20200806-covid-19 sitrep-199.pdf?sfvrsn=6b9d262d_2.

[11] World Health Organization, WHO Director-General's Opening Remarks at the Media Briefing on COVID-19-3 August 2020, World Health Organization, Geneva, Switzerland, 2020, https://www.who.int/dg/speeches/detail/whodirector-general-s-opening-remarks-at-the-media-briefingon-covid-19---3-august-2020.

[12] National Health Commission of the People's Republic of China, Guidelines for the Diagnosis and Treatment of COVID19 Pneumonia, National Health Commission of the People's Republic of China, Beijing, China, 2020, http://www.nhc.gov. $\mathrm{cn} /$.

[13] D. Moher, K. F. Schulz, and D. Altman, "The CONSORT statement: revised recommendations for improving the quality of reports of parallel-group randomized trials," JAMA, vol. 285, no. 15, pp. 1987-1991, 2001.

[14] A. R. Jadad, R. A. Moore, D. Carroll et al., "Assessing the quality of reports of randomized clinical trials: is blinding necessary?" Controlled Clinical Trials, vol. 17, no. 1, pp. 1-12, 1996.

[15] J. P. T. Higgins, D. G. Altman, P. C. Gotzsche et al., "The cochrane collaboration's tool for assessing risk of bias in randomised trials," BMJ, vol. 343, p. d5928, 2011.

[16] J. P. T. Higgins, S. G. Thompson, J. J. Deeks, and D. G. Altman, "Measuring inconsistency in meta-analyses," BMJ, vol. 327, no. 7414, pp. 557-560, 2003.

[17] R. DerSimonian and N. Laird, "Meta-analysis in clinical trials," Controlled Clinical Trials, vol. 7, no. 3, pp. 177-188, 1986.

[18] S. Duval and R. Tweedie, "Trim and fill: a simple funnel-plotbased method of testing and adjusting for publication bias in meta-analysis," Biometrics, vol. 56, no. 2, pp. 455-463, 2000.

[19] Y. Feng, "Observation of clinical effect of Lianhuaqingwen capsule combined with cefuroxime in the treatment of community-acquired pneumonia," Renowned Doctor, no. 1, p. 236, 2020.

[20] Y. Wang, J. W. Peng, and T. Tan, "Effects of lianhuaqingwen granules on pulmonary function and serum inflammatory factors in children with mycoplasma pneumonia pneumonia," Journal of Pediatric Pharmacy, vol. 25, no. 4, pp. 35-37, 2019.

[21] Q. Lin, C. S. Liao, X. H. Wei et al., "Therapeutic effect of Lianhua Qingwen granule combined with sequential therapy of azithromycin on children mycoplasma pneumoniae pneumonia complicated with atelectasis and its effect on T lymphocyte subsets and inflammatory factors," Modern Journal of Integrated Traditional Chinese and Western Medicine, vol. 28, no. 2, pp. 153-158, 2019.

[22] D. D. Sun, "Evaluation of the efficacy and safety of Lianhuaqingwen granule combined with cefoperazone sodiumsulbactam sodium in patients with community-acquired pneumonia," Anti-Infection Pharmacy, vol. 16, no. 8, pp. 1423-1425, 2019.

[23] N. Xia and F. Han, "Analysis of the application of Lianhuaqingwen and oseltamivir phosphate capsules in the treatment of pneumonia," China Health Care \& Nutrition, vol. 29, no. 16, p. 280, 2019.

[24] X. Q. Hu, Y. Wan, Q. Lu et al., "Clinical study of Lianhuaqingwen capsules combined with cefuroxime in treatment of community acquired pneumonia," Drugs \& Clinic, vol. 33, no. 12, pp. 3216-3220, 2018.

[25] H. Lv, Z. N. Ouyang, J. Wang et al., "Clinical study on Lianhua Qingwen capsules combined with levofloxacisn in treatment of pneumonia,” Drugs \& Clinic, vol. 33, no. 9, pp. 2294-2297, 2018.

[26] T. Y. Chen, S. Zhu, H. E. Bian et al., "Effect of Lianhua Qingwen capsules on pulmonary function index, serum inflammatory factor level and curative effect in children with mycoplasma pneumoniae pneumonia," Chinese Archives of Traditional Chinese Medicine, vol. 36, no. 11, pp. 2713-2715, 2018.

[27] J. P. Zhang, "Observation of the effect of Lianhua Qingwen granule combined with moxifloxacin tablets on elderly community-acquired pneumonia," Chinese Journal of Health Care Nutrition, vol. 28, no. 27, p. 235, 2018.

[28] L. Zhang and L. Han, "Clinical observation of Lianhua Qingwen capsules combined with piperacillin and sulbactam in treatment of pneumonia," Drugs \& Clinic, vol. 33, no. 8, pp. 1960-1963, 2018.

[29] C. Liu and H. A. Wang, "Clinical study on Lianhua Qingwen capsules combined with cefoperazone sodium and sulbactam sodium in treatment of community acquired pneumonia," Drugs \& Clinic, vol. 32, no. 7, pp. 1251-1254, 2017.

[30] C. Y. Han, "Effect of lianhuaqingwen granule on the cellular immune function of mycoplasma pneumoniae pneumonia in children," Asia-Pacific Traditional Medicine, vol. 13, no. 5, pp. 137-138, 2017.

[31] Y. P. Yang, "Observation of therapeutic effect of Lianhua Qingwen granule on community-acquired pneumonia," Chinese Journal of Clinical Rational Drug Use, vol. 9, no. 6A, pp. 71-72, 2016.

[32] X. F. Tu, L. X. Hu, Q. Z. Peng et al., "Influence of Lianhua Qingwen granules to cellular immune function in children with mycoplasma pneumonia," Journal of Practical Medical Techniques, vol. 22, no. 12, pp. 1263-1265, 2015.

[33] G. S. Wang and L. X. Zhao, "Clinical observation of Lianhua Qingwen capsule in elderly community acquired pneumonia with fever," Oriental Diet Therapy and Health Care, vol. 5, p. 38, 2015.

[34] X. R. Xu, Q. Jin, W. Xu et al., "Clinical observation of Lianhua Qingwen granule in adjuvant treatment of community acquired pneumonia with heat toxicity," Zhejiang Clinical Medical Journal, vol. 17, no. 10, pp. 1729-1730, 2015.

[35] X. H. Meng, L. Q. Li, and L. M. Wang, "Analysis of the effect of Lianhua Qingwen granule in treating uremic complicated pneumonia," Contemporary Medicine Forum, vol. 13, no. 17, pp. 30-31, 2015.

[36] J. Zhu, H. Q. Luo, and M. F. Sheng, "Comparison of Lianhua Qingwen capsule combined with western medicine and simple western medicine in the treatment of pulmonary infection," Liaoning Journal of Traditional Chinese Medicine, vol. 42, no. 4, pp. 797-798, 2015.

[37] X. Li, F. Jia, and M. Peng, "Observation on the therapeutic effect of Lianhua Qingwen capsule combined with azithromycin on community-acquired pneumonia," Chinese Journal of Clinical Rational Drug Use, vol. 7, no. 12, pp. 134-135, 2014.

[38] Y. C. Li, "Clinical observation on treatment of 49 cases of viral pneumonia with Lianhua Qingwen capsule," Chinese Journal of Difficult and Complicated Cases, pp. 691-692, 2014.

[39] W. B. Liu, X. J. Ding, and L. M. Lou, "Therapeutic effect of Lianhua Qingwen granules on community acquired pneumonia," Chinese Journal of Difficult and Complicated Cases, pp. 693-695, 2014.

[40] X. Jiang, Z. Wang, T. Yu et al., "Clinical observation of Lianhua Qingwen capsule in treating community acquired 
pneumonia," Chinese Journal of Clinical Rational Drug Use, vol. 7, no. 10, pp. 59-60, 2014.

[41] S. G. Gao, "Observation on the efficacy and safety of Lianhua Qingwen granule in adjuvant treatment of pneumonia," Journal of Modern Medicine \& Health, vol. 30, no. 12, pp. 1876-1877, 2014.

[42] H. X. Zou, "Clinical effect of Lianhua Qingwen granules combined with moxifloxacin tablets in the treatment of acquired pneumonia in the elderly community," Medical Aesthetics and Cosmetology, no. 7, pp. 203-204, 2014.

[43] F. L. Dong, "Observation of curative effect of Lianhua Qingwen granules combined ceftriaxone sodium for injection on the treatment of senile bacterial pneumonia," Hebei Journal of Traditional Chinese Medicine, vol. 36, no. 10, pp. 1530-1531, 2014.

[44] D. P. Wang and W. Huang, "Observation on the therapeutic effect of Lianhua Qingwen granule on non-severe community-acquired pneumonia," Journal of Tropical Diseases and Parasitology, vol. 12, no. 3, pp. 176-177, 2014.

[45] J. Wang, "Therapeutic effect observation of Lianhua Qingwen particles in treating patients with bronchopneumonia," Chinese Journal of Difficult and Complicated Cases, pp. 703-704, 2014.

[46] S. J. Zhou and Q. Fang, "Clinical evaluation of Lianhua Qingwen particle on treatment of pneumonia," Journal of Difficult Diseases, vol. 48, pp. 700-702, 2014.

[47] W. H. Wang, "The observation of curative effect of Lianhua Qingwen capsule for 100 patients with upper respiratory tract infection and pneumonia observation," Journal of Taishan Medical College, vol. 34, no. 2, pp. 128-130, 2013.

[48] X. D. Zhong, X. Q. Chen, and J. R. Wu, "Observation on 38 cases of community acquired pneumonia treated by Lianhua Qingwen capsule," Zhejiang Journal of Traditional Chinese Medicine, vol. 48, no. 5, p. 385, 2013.

[49] B. Chen, Y. W. Yao, and H. Q. Wang, "Efficacy of Lianhua Qingwen granule combined with moxifloxacin tablets in the treatment of elderly community-acquired pneumonia $\mathrm{Ob}$ served," Jilin Medical Journal, vol. 34, no. 1, pp. 79-80, 2013.

[50] H. X. Peng, "The rearch on clinical efficacy of facilitating the flow of lung-qi and ciear lung-heat," in Treatment of Community-acquired Pneumonia (Lung-Heat Syndrome)Guangzhou University of Chinese Medicine, Guangzhou, China, 2013.

[51] Q. Xu, N. Zhang, W. S. Yuan et al., "Observation on the therapeutic effect of Lianhua Qingwen capsule on community-acquired pneumonia," Journal of Emergency in Traditional Chinese Medicine, vol. 21, no. 8, pp. 1299-1300, 2012.

[52] D. Z. Cheng and Y. Li, "Clinical effectiveness and case analysis in 54 NCP patients treated with Lianhua Qingwen granules," World Chinese Medicine, vol. 15, no. 2, pp. 150-154, 2020.

[53] R. B. Lv, W. J. Wang, and X. Li, "Clinical observation on 63 suspected cases of new coronavirus pneumonia treated by lianhua qingwen granule combined with western medicine conventional therapy," Journal of Traditional Chinese Medicine, vol. 61, no. 8, pp. 655-659, 2020.

[54] K. T. Yao, M. Y. Liu, X. Li et al., "Retrospective clinical analysis on treatment of novel coronavirus-infected pneumonia with traditional Chinese medicine lianhua qingwen," Chinese Journal of Experimental Traditional Medical Formulae, vol. 26, no. 11, pp. 8-12, 2020.

[55] Y. Chen, C. Q. Han, F. L. Zhao et al., "Effects of lianhua qingwen granules combined with azithromycin on children with mycoplasma pneumoniae pneumonia," World Chinese Medicine, vol. 15, no. 1, pp. 76-80, 2020.
[56] L. Q. Chen and W. J. Chen, "Lianhua Qingwen granules for mycoplasma pneumoniae in children," China Health Standard Management, vol. 11, no. 11, pp. 104-106, 2020.

[57] K. Hu, W.-j. Guan, Y. Bi et al., "Efficacy and safety of Lianhua Qingwen capsules, a repurposed Chinese herb, in patients with coronavirus disease 2019: a multicenter, prospective, randomized controlled trial," Phytomedicine, Article ID 153242, 2020.

[58] P. Yu, Y. Z. Li, S. B. Wan et al., "Efficacy of Lianhua Qingwen combined with arbidol in the treatment of mild COVID-19 pneumonia," Chinese Pharmaceutical Journal, pp. 1-9, 2020.

[59] W. G. Xia, C. Q. An, C. J. Zheng et al., "A clinical study on 34 cases of COVID-19 pneumonia treated with combination of traditional Chinese and western medicines," Journal of Traditional Chinese Medicine, vol. 61, no. 5, pp. 375-382, 2020.

[60] J. Shi, Z. G. Yang, C. Ye et al., "Clinical observation on 49 cases of non-critical COVID-19 in Shanghai treated by integrated traditional Chinese and western medicine," Shanghai Journal of Traditional Chinese Medicine, vol. 54, no. 4, pp. 30-35, 2020.

[61] P. T. Nguyen, H. T. Tran, D. A. Fitzgerald et al., "Antibiotic use in children hospitalised with pneumonia in Central Vietnam," Archives of Disease in Childhood, vol. 105, no. 8, pp. 713-719, 2020.

[62] G. K. Møller, J. J. Nygaard, L. Bjerrum et al., "Short-course vs long-course antibiotic treatment for community-acquired pneumonia: a literature review," Basic \& Clinical Pharmacology and Toxicology, vol. 124, no. 5, pp. 550-559, 2019.

[63] O. N. Jim, "Tackling drug-resistant infections globally: report and recommendations," 2020, https://www.linkedin.com/ pulse/tackling-drug-resistant-infections-globally-reportpooja-bheda.

[64] W. W. Cui, C. Wei, and H. T. Wong, "The progress of Lianhua Qingwen capsule on respiratory system disease," Basic and Clinical Research of Collateral Disease, vol. 10, pp. 657-659, 2014.

[65] L. Lin, F. Dai, G. Ren, J. Wei, Z. Chen, and X. Tang, "Efficacy of Lianhua Qingwen granules in the management of chronic rhinosinusitis without nasal polyps," American Journal of Otolaryngology, vol. 41, no. 1, Article ID 102311, 2020.

[66] H. Guo, Q. H. Zhang, J. Yang et al., "Effect of lianhua qingwen on immunity of mice infected with flu virus," Journal of Nanjing TCM University, vol. 23, no. 2, pp. 106-108, 2007.

[67] L. Runfeng, H. Yunlong, H. Jicheng et al., "Lianhua Qingwen exerts anti-viral and anti-inflammatory activity against novel coronavirus (SARS-CoV-2)," Pharmacological Research, vol. 156, Article ID 104761, 2020.

[68] W. W. Cui, X. Jin, Y. F. Zhang et al., "Effect of Lianhua Qingwen capsules on inflammatory cytokines and junction protein expression in mice with acute lung injury induced by lipopolysaccharides," Chinese Journal of Pharmacology and Toxicology, vol. 29, no. 2, pp. 213-219, 2015.

[69] D. Gao, M. Niu, S. Z. Wei et al., "Identification of a pharmacological biomarker for the bioassay-based quality control of a thirteen-component TCM formula (Lianhua Qingwen) used in treating influenza A virus (H1N1) infection," Frontiers in Pharmacology, vol. 11, p. 746, 2020.

[70] G. Li and E. De Clercq, "Therapeutic options for the 2019 novel coronavirus (2019-nCoV)," Nature Reviews Drug Discovery, vol. 19, no. 3, pp. 149-150, 2020.

[71] J. Liu, E. Manheimer, Y. Shi, and C. Gluud, "Chinese herbal medicine for severe acute respiratory syndrome: a systematic review and meta-analysis," The Journal of Alternative and Complementary Medicine, vol. 10, no. 6, pp. 1041-1051, 2004. 
[72] T. Li and T. Peng, "Traditional Chinese herbal medicine as a source of molecules with antiviral activity," Antiviral Research, vol. 97, no. 1, pp. 1-9, 2013.

[73] Y. Yang, M. S. Islam, J. Wang, Y. Li, and X. Chen, "Traditional Chinese medicine in the treatment of patients infected with 2019-new coronavirus (SARS-CoV-2): a review and perspective," International Journal of Biological Sciences, vol. 16, no. 10, pp. 1708-1717, 2020.

[74] H. Lu, C. W. Stratton, and Y. W. Tang, "Outbreak of pneumonia of unknown etiology in Wuhan, China: the mystery and the miracle," Journal of Medical Virology, vol. 92, no. 4, pp. 401-402, 2020.

[75] Z. Zhou, S. M. Wang, and Y. P. Liu, "A meta-analysis of the effect and safety of Lianhua Qingwen capsules in the treatment of community acquired pneumonia," China Medical Herald, vol. 14, no. 33, pp. 133-137, 2017.

[76] M. Liu, Y. Gao, Y. Yuan et al., "Efficacy and safety of integrated traditional Chinese and western medicine for corona virus disease 2019 (COVID-19): a systematic review and meta-analysis," Pharmacological Research, vol. 158, Article ID 104896, 2020.

[77] Y. Pharmaceutical, "Announcement of Shijiazhuang Yiling pharmaceutical Co., Ltd. on media reports,” 2020, http:// finance.ifeng.com/c/7wObPca8OSa.

[78] Y. X. Wang, K. Y. Zhang, J. H. Huang et al., "Clinical drug safety of Lianhua Qingwen preparation: a systematic evaluation," Evaluation and Analysis of Drug-use in Hospitals of China, vol. 13, no. 8, pp. 676-681, 2013.

[79] S. H. Wang, J. F. Liu, Y. L. Zhang et al., "Systematic review of efficacy and safety of Lianhua Qingwen capsules in treatment of viral influenza," Zhongguo Zhong Yao Za Zhi, vol. 44, no. 7, pp. 1503-1508, 2019.

[80] Q. Q. Niu, Y. Chen, Y. Liu et al., "Efficacy and safety of Lianhua Qingwen capsule for influenza: a systematic review," Zhongguo Zhong Yao Za Zhi, vol. 42, no. 8, pp. 1474-1481, 2017.

[81] P. Zhao, H. Z. Yang, H. Y. Lv et al., "Efficacy of Lianhua Qingwen capsule compared with oseltamivir for influenza A virus infection: a meta-analysis of randomized, controlled trials," Alternative Therapies in Health and Medicine, vol. 20, no. 2, pp. 25-30, 2014.

[82] X. H. Peng, The Research on Clinical Efficacy of Facilitating the Flow of Lung-qi and Clear Lung-Heart of Community-Acquired Pneumonia (Lung-Heart Syndrome), Traditional Chinese Medicine University of Guangzhou, Guangzhou, China, 2013. 